\title{
Brasil, 1981-2013: efectos del crecimiento económico y de la desigualdad de los ingresos en la pobreza
}

\author{
Francisco José Silva Tabosa, Pablo Urano \\ de Carvalho Castelar y Guilherme Irffi
}

\section{Resumen}

En este artículo se analizan los efectos del crecimiento económico y la desigualdad de los ingresos en la pobreza del Brasil entre 1981 y 2013. Para ello se utiliza un modelo de panel dinámico, mediante el método generalizado de momentos de dos pasos desarrollado por Blundell y Bond (1998) para tres períodos. El primero, que comprende a los otros dos, va de 1981 a 2013, mientras que el segundo y el tercero corresponden a las etapas anterior y posterior a la implementación del Plan Real (1981-1994 y 1995-2013, respectivamente). Los resultados muestran que, para combatir la pobreza en el Brasil, las políticas de crecimiento económico que promueven simultáneamente el aumento de los ingresos y la reducción de las disparidades son preferibles a las que priorizan solo el aumento de los ingresos medios. Se observa también un crecimiento a favor de los pobres en el período posterior al Plan Real.

Palabras clave

Crecimiento económico, distribución del ingreso, pobreza, medición, modelos econométricos, mitigación de la pobreza, historia económica, Brasil

Clasificación JEL

O15, C32, C22

Autores

Francisco José Silva Tabosa es Profesor del Departamento de Economía Agrícola de la Universidad Federal de Ceará, Brasil. franzetabosa@ufc.br

Pablo Urano de Carvalho Castelar es Profesor del Curso de Finanzas en la Facultad de Economía, Administración, Actuaría y Contabilidad de la Universidad Federal de Ceará, Brasil. pcastelar@ufc.br

Guilherme Irffi es Profesor del Departamento de Economía Aplicada de la Facultad de Economía, Administración, Actuaría y Contabilidad de la Universidad Federal de Ceará, Brasil.guidirffi@gmail.com 


\section{Introducción}

La reducción de la pobreza se relaciona estrechamente con la desigualdad de los ingresos y con los ingresos medios de un país o una región (Bourguignon, 2002). Por ese motivo, es oportuno determinar el tipo de política más eficaz para invertir las situaciones de pobreza con mayor rapidez. En otras palabras, ¿para reducir la pobreza es necesario promover políticas destinadas al incremento de los ingresos medios o a la reducción de la desigualdad?

Ante esa pregunta, en los últimos años se presentaron y destacaron diversos estudios sobre los efectos de las variaciones en los ingresos y la desigualdad en la reducción de la pobreza, pues en varios países (y regiones) el crecimiento económico por sí solo no ha resuelto este problema (Ravallion, 1997; Rocha, 2006).

Debido a que la variación en los niveles de pobreza puede obedecer a la redistribución de los ingresos o al crecimiento económico (o ambos), cabe ponderar la importancia de cada efecto de esas variables en la pobreza (Ravallion, 1997). En virtud de esos resultados, los investigadores han buscado determinar los factores que influyen en la reducción de la pobreza.

Ravallion y Chen (1997), por ejemplo, estimaron la elasticidad ingreso de la pobreza (medida por el número de personas con ingresos por debajo de la línea de pobreza de 1 dólar por día) en una muestra de países en desarrollo y obtuvieron un valor de -3. Esto significa que por cada aumento del $1 \%$ de los ingresos medios la proporción de personas con ingresos por debajo de la línea de pobreza se reduce un $3 \%$.

Sin embargo, no hay consenso hasta ahora con respecto a la naturaleza de las relaciones entre pobreza, crecimiento y desigualdad de los ingresos. De acuerdo con Barreto (2005), gran parte de la evidencia empírica parece indicar que en los países con bajos ingresos y poca desigualdad se respondería de forma más contundente a las políticas de crecimiento económico, mientras que sus efectos serían menores en las economías de mayores ingresos y mayor desigualdad, donde las políticas de reducción de la disparidad serían más eficaces.

Por otra parte, en una serie de trabajos en los que se analiza el caso del Brasil ${ }^{1}$, se muestra que la pobreza es más sensible a las políticas orientadas a reducir la concentración de los ingresos que a las que procuran aumentar los ingresos medios. Por esa razón, es extremadamente importante determinar los efectos que cada uno de esos factores tiene sobre el otro, para contribuir al debate sobre las políticas públicas más eficaces para la reducción de la pobreza y la desigualdad.

En el trabajo de Barros y otros (2007) se verifica que la incidencia de la pobreza es mayor en el Brasil que en la mayoría de los países con ingresos per cápita similares y se constata que la desigualdad de los ingresos es responsable de que el crecimiento económico sea relativamente ineficaz en la reducción de la pobreza. Esto significaría que el efecto del crecimiento económico en la reducción de la pobreza es menor en el Brasil que en otros países que alcanzaron el mismo nivel de ingresos.

De acuerdo con Rodrik (2000), las políticas adoptadas para esta finalidad se concentran en estimular el crecimiento económico, en el sentido de que el aumento de los ingresos medios de la economía, la reducción de la desigualdad de los ingresos entre las personas o ambos puedan reducir los índices de pobreza. Sin embargo, es imprescindible evaluar el peso que se debe dar a cada una de esas estrategias, tanto a nivel de las regiones como de los estados.

En este contexto, el objetivo de este artículo es analizar los efectos del crecimiento económico y de la desigualdad de los ingresos en los índices de pobreza en el Brasil en el período de 1981 a 2013, por medio de modelos para datos de panel. La estimación de datos agrupados por mínimos

1 Véanse, por ejemplo, Hoffmann (2005), De Lima, Barreto y Marinho (2003) y Menezes y Pinto (2005). 
cuadrados ordinarios (MCO), con el método dentro de los grupos (Within Groups) y mediante el System-GMM, un modelo de panel dinámico, estimado por el método generalizado de momentos (MGM) de dos pasos, desarrollado por Blundell-Bond (1998), permite examinar y estimar la persistencia en los índices de pobreza en el Brasil.

El principal aporte de este trabajo consiste en estimar parámetros de modelos relativos a los subperíodos de 1981 a 1994 y de 1995 a 2013, para analizar las etapas anterior y posterior al Plan Real, con miras a comparar las elasticidades ingreso y desigualdad de la pobreza y de la pobreza extrema en distintos períodos de la economía brasileña.

Para alcanzar esos objetivos, el estudio se divide en cinco secciones, incluida esta Introducción. En la segunda se presenta una breve revisión de la literatura sobre las relaciones entre el crecimiento económico y la desigualdad de los ingresos con la pobreza. A continuación se presentan la base de datos, los modelos y la metodología econométrica aplicada. El análisis y la discusión de los resultados obtenidos en la estimación de los modelos se detallan en la cuarta sección. Por último, se realizan algunas consideraciones finales.

\section{Revisión de la literatura: la relación triangular entre pobreza, crecimiento económico y desigualdad}

De acuerdo con Bourguignon (2002), existe una relación triangular entre pobreza, crecimiento económico y desigualdad de los ingresos, es decir, una interacción entre esas tres variables, que proporciona las condiciones necesarias para diagnosticar la medida en que el aumento de los ingresos o la reducción de la desigualdad incidirían en la reducción de la pobreza. En consecuencia, se optó por presentar la evidencia empírica sobre el tema en tres secciones.

La primera se reserva a los estudios en los que se analiza la relación entre pobreza y crecimiento económico, mientras que la segunda se dedica a los análisis de la relación entre pobreza y desigualdad y la última a la literatura sobre la relación entre crecimiento económico y desigualdad de los ingresos.

\section{Pobreza y crecimiento económico}

En la literatura empírica nacional e internacional orientada al estudio de las relaciones entre crecimiento económico y pobreza se utilizan dos opciones para representar el crecimiento económico: el producto interno bruto (PIB) o los ingresos medios. En esos estudios hay cierta convergencia entre los resultados en cuanto a que para reducir la pobreza, como destacan Araújo, Tabosa y Khan (2012), existen dos factores fundamentales: la tasa media de crecimiento y el nivel inicial de la desigualdad de los ingresos.

La relación entre el crecimiento económico y la reducción de la pobreza puede medirse mediante la elasticidad ingreso o elasticidad crecimiento. Si esta es elevada, las políticas públicas de combate a la pobreza basadas en el crecimiento económico son más eficaces, pues el aumento de los ingresos lleva a la reducción de la pobreza en mayor proporción. En el caso contrario, si dicha elasticidad es baja, las estrategias de reducción de la pobreza más adecuadas deberían combinar el crecimiento económico con algún tipo de redistribución de los ingresos (Marinho y Araújo, 2010).

Ravallion y Chen (1997) estimaron las elasticidades ingreso-pobreza e ingreso-desigualdad sobre la base de datos de 45 países. Sus resultados mostraron que si los ingresos aumentaran un $1 \%$ en los países con bajos niveles de desigualdad, la pobreza disminuiría un 4,3\%, mientras que en los países donde la desigualdad es elevada, la disminución de la pobreza sería del 0,6\%. En este último 
caso, concluyeron que el crecimiento presenta un efecto reducido en la pobreza. Sin embargo, si la desigualdad disminuye a raíz del crecimiento, la reducción de la pobreza es satisfactoria.

Chen y Wang (2001) estudiaron la relación entre pobreza, ingresos y desigualdad en China en los años noventa y llegaron a la conclusión de que la pobreza se redujo por el crecimiento económico y la concentración de los ingresos contribuyó a aumentarla.

En un contexto más reciente, Figueiredo y Laurini (2015) realizaron un análisis internacional con respecto a la elasticidad de la pobreza. La propuesta del trabajo es aplicar un método no paramétrico a un panel de países, utilizando la base de datos PovcalNet del Banco Mundial, que incluye 139 observaciones relativas a 93 países en desarrollo. La principal preocupación de los autores es corregir posibles problemas de endogeneidad, que pueden surgir por una serie de factores, como problemas de simultaneidad, factores financieros que afectan a los componentes analizados de la misma manera, o la hipótesis comúnmente adoptada de la forma funcional de la relación entre crecimiento, pobreza y desigualdad, que se asume es una relación lineal, pero que de acuerdo con algunos indicios en la literatura no lo sería.

Los autores argumentan que otro de los aportes del trabajo consiste en determinar los efectos directos e indirectos del crecimiento en la pobreza y en la desigualdad con la mencionada metodología no paramétrica. De acuerdo con los resultados del estudio, la metodología generalmente adoptada en los trabajos empíricos que analizan el crecimiento, la pobreza y la desigualdad suele sobrestimar los efectos del crecimiento en la pobreza. Así, la influencia de las crisis o los períodos de expansión económica en la pobreza sería menor de la que generalmente se les atribuye.

En el ámbito nacional, teniendo en cuenta los datos de 26 estados brasileños relativos al período de 1985 a 1999 y utilizando un procedimiento metodológico que permitió descomponer la variación en la pobreza derivada de cambios en los ingresos medios y modificaciones en la concentración de los ingresos (medida por el coeficiente de Gini), Marinho y Soares (2003) encuentran pruebas de la eficacia del efecto crecimiento en la reducción de la pobreza, sobre todo en los estados del norte del Brasil. En ese sentido, el incremento de los ingresos ha sido la estrategia preferida para combatir la pobreza.

En otro trabajo sobre esa problemática en el Brasil, Hoffmann (2005), sobre la base de datos de la Encuesta Nacional de Hogares (PNAD) de 1999, encuentra que un aumento del 1\% en el ingreso per cápita del hogar en el Brasil lleva a una reducción del 0,84\% en la proporción de pobres, y que el valor absoluto de esa elasticidad crece con el ingreso y disminuye con el aumento de la desigualdad.

Manso, Barreto y Tebaldi (2006) utilizaron datos de la PNAD de 1995 a 2004 para tratar de evidenciar las relaciones entre el crecimiento de los ingresos, la reducción de la pobreza y el perfil distributivo de la riqueza. De acuerdo con esos autores, los componentes de crecimiento de los ingresos medios y de distribución de los ingresos son suficientes para explicar gran parte de las variaciones en los niveles de pobreza entre los estados brasileños. Los resultados de esos trabajos demuestran que las políticas de lucha contra la pobreza mediante el crecimiento son más eficaces cuando se combinan con la redistribución de los ingresos (Barreto, 2005).

Otro punto que se ha destacado en los estudios sobre la relación entre la pobreza y el crecimiento económico es la importancia de determinar si el crecimiento es a favor de los pobres o no. Ravallion (2004) indica dos corrientes de crecimiento a favor de los pobres: i) una reducción de la pobreza mayor que la que se produciría si todos los ingresos hubieran aumentado en la misma proporción (Kakwani y Pernia, 2000); ii) el crecimiento que reduce la pobreza, independientemente de su proporción (Ravallion y Chen, 2003).

En el primer caso, el análisis puede realizarse mediante la elasticidad ingreso de la pobreza, pues si el valor de dicha elasticidad es superior a 1 (en términos absolutos), significa que la reducción de la pobreza fue superior al crecimiento de los ingresos (Kakwani y Pernia, 2000). En el segundo caso, independientemente del valor de la elasticidad ingreso de la pobreza, si el aumento de los 
ingresos se traduce en una reducción de la pobreza, ya constituye un crecimiento a favor de los pobres (Ravallion, 2004).

El trabajo de França (2010), que estudia el tema del crecimiento a favor de los pobres y sus efectos regionales en el Brasil, es un ejemplo específico de este tipo de análisis y tiene un objetivo similar al que se propone en este artículo. Los resultados obtenidos con la metodología de Kakwani, Khandker y Son (2004), sobre la base de datos de las regiones brasileñas de 1995 a 2005, indican que los efectos de las políticas contra la pobreza son mayores en las regiones sur y sudeste del país. Posteriormente se calculan las elasticidades ingreso-pobreza y desigualdad-pobreza y se observa que como esta última es mayor que la primera, la reducción de la desigualdad medida por el coeficiente de Gini es más sensible a la reducción de los niveles de desigualdad que al incremento de los ingresos medios. El autor utiliza la metodología de Kraay (2004) para analizar la importancia de cada componente del crecimiento a favor de los pobres y observa que, en el nordeste del país, el aumento de los ingresos ha sido relativamente más importante que la desigualdad como fuente de crecimiento a favor de los pobres.

Se destaca también el estudio realizado por Kakwani, Neri y Son (2010), que utilizaron datos de la PNAD relativos al período de 1995 a 2004 y, sobre la base de una línea de pobreza que tenía en cuenta los costos de vida regionales ${ }^{2}$ y la evolución de los indicadores sociales basados en los ingresos medios per cápita, detectaron la presencia de crecimiento a favor de los pobres en el Brasil en el período posterior a la implementación del Plan Real.

Netto Júnior y Figueiredo (2014) aplicaron un enfoque no paramétrico para analizar el crecimiento a favor de los pobres en el Brasil y sus principales regiones geográficas y estados en los períodos 1987-1993, 1993-1999 y 1999-2007. Los resultados sugieren que en todos ellos hubo crecimiento a favor de los pobres en el período 1987-2007.

\section{Pobreza y desigualdad}

La desigualdad de los ingresos es un componente importante en el debate sobre la pobreza. La pobreza es un problema mundial, que en algunos países persiste a pesar de las crecientes reservas de riqueza material. Su extensión y gravedad quedan demostradas por el número de personas en situación de pobreza en todos los países.

De hecho, se observa una relación intrínseca entre desigualdad y pobreza. Conforme Bourguignon (2002) y Ravallion (1997), la reducción de la desigualdad de los ingresos es un instrumento importante para la reducción de la pobreza, mientras que el crecimiento económico puede no ser un elemento tan necesario. Así, la reducción de la desigualdad puede desempeñar un papel crucial en la transformación del crecimiento para la reducción de la pobreza.

Banerjee y Duflo (2003) subrayan que el crecimiento económico tiene una relación no lineal con la desigualdad, es decir, que la desigualdad tiene efectos directos e indirectos en la pobreza. Figueiredo y Laurini (2015) procuraron medir tales efectos utilizando un panel de 93 países. Los autores encontraron que la asociación no lineal entre crecimiento y desigualdad parece corroborarse, que por lo general no se presta suficiente atención a dicha relación y que esta tiene un vínculo positivo con la pobreza.

También en ese sentido, la reducción de la pobreza puede alcanzarse rápidamente cuando un país en crecimiento presenta una distribución menos desigual de los ingresos (Barreto, 2005). Por lo tanto, además de resolver el problema específico, la implementación de políticas públicas para la reducción de la desigualdad también puede alcanzar indirectamente otras metas de política

2 Véanse más detalles en Kakwani, Neri y Son (2010) y Ferreira, Lanjouw y Neri (2003). 
económica, como el aumento del crecimiento y la reducción de la pobreza. La pobreza presenta mayor sensibilidad con respecto a la desigualdad que al crecimiento (Marinho y Soares, 2003). En consecuencia, las políticas destinadas a reducir la pobreza deben privilegiar medidas para disminuir la concentración de los ingresos.

De acuerdo con Rocha (2006), la proporción de pobres en el Brasil se redujo alrededor de dos puntos porcentuales en el período de 2001 a 2004 a raíz de diversos factores, cuyos efectos varían entre las regiones, en las cuales se observan cambios distributivos en el ingreso del trabajo y la expansión de las prestaciones sociales. Para la autora, la persistencia de la pobreza en el Brasil se debe en gran parte a la desigualdad existente. Rocha sostiene que la pobreza puede reducirse, tanto mediante el incremento de los ingresos como por la mejora de su distribución, y que se debe hacer hincapié en la reducción de la desigualdad de los ingresos, porque el incremento de los ingresos sin reducción de la desigualdad significa transferir a un horizonte futuro la eliminación de la pobreza en el país.

Sin embargo, una gran desigualdad inicial reduce los efectos del crecimiento económico en la pobreza. Al examinar los trabajos sobre crecimiento y desigualdad, Ferreira (1999) concluye que no hay consenso acerca de que el crecimiento resulte afectado por los niveles iniciales de desigualdad de los ingresos.

En las últimas dos décadas, se ha registrado una baja reducción o incluso un aumento de la pobreza en un número considerable de países. Para Fosu (2010), ese decepcionante desempeño puede atribuirse en parte a la concentración del crecimiento, sumada a la elevada y creciente desigualdad de los ingresos en muchos países de América Latina.

\section{Crecimiento y desigualdad}

La relación entre el crecimiento económico y la desigualdad se ha analizado en la literatura teniendo en cuenta las causalidades existentes entre esas variables. Se han debatido muchos temas relacionados con ellas, que van desde las causas de la desigualdad y la manera en que se perpetúa a lo largo del tiempo a la relación entre la desigualdad y el proceso de desarrollo económico. Para Diniz (2005), entre esas dos variables existe una relación de doble causalidad.

La hipótesis de Kuznets (1955) de la "U invertida" constituye el punto de partida de esa vertiente. Se considera que, en primer lugar, la desigualdad aumentaría con el inicio del desarrollo económico, que tiene lugar cuando la economía pasa del modelo rural a la industrialización (transferencia de la forma de trabajo del sector menos productivo al más productivo). Más adelante, la desigualdad disminuiría cuando la mayor parte de la mano de obra se empleara en el sector más productivo. En consecuencia, la política de desarrollo podría limitarse a la promoción del crecimiento económico y este, en última instancia, promovería la reducción de la desigualdad. En un estudio comparado entre 98 países, Barro (2000) mostró que la curva de Kuznets presenta cierta regularidad empírica a lo largo del tiempo, pero explica relativamente poco la variación de la desigualdad entre países.

Para algunos autores, la desigualdad puede ser perjudicial para el crecimiento económico. Como se resume en Castelar (2007), en estudios empíricos realizados sobre todo a partir de 1996 se observa que la desigualdad inicial afecta negativamente el crecimiento económico posterior. En la mayor parte de la literatura sobre el tema se indica que la reducción de una unidad en la desviación estándar de la desigualdad aumenta la tasa anual de crecimiento del PIB per cápita de 0,5 a 0,8 puntos porcentuales. Sin embargo, al utilizar datos en panel se observa una relación más débil entre la desigualdad de los ingresos y el crecimiento económico, que lleva a creer que la regularidad empírica, expresada por la curva de Kuznets, es robusta en datos de corte transversal, pero desaparece cuando se introducen efectos fijos de países. Entre los ejemplos de tales trabajos cabe mencionar Fields y Jakubson (1994), Fishlow (1972), Deineger y Squire (1998) y Barreto, Melo Neto y Tebaldi (2001). 
De acuerdo con Stewart (2000) y Fosu (2010), una elevada desigualdad de los ingresos da lugar a inestabilidad política, incertidumbre, menor inversión, bajos niveles de crecimiento y políticas tributarias redistributivas populistas. Los efectos de disuasión y la mayor desigualdad influyen en los grupos más ricos, que presionan por un tratamiento tributario preferencial. Esto se traduce en el exceso de inversiones en determinadas áreas y la reducción del crecimiento.

Easterly (2000) y Adams (2004) analizaron las relaciones entre crecimiento y desigualdad de los ingresos entre países y observaron que la elasticidad crecimiento de la pobreza es mayor en el grupo con los coeficientes de Gini más bajos (menor desigualdad). Los autores destacan la importancia de la reducción de la desigualdad en la determinación de la capacidad de respuesta de la pobreza al crecimiento de los ingresos.

En contrapartida, en trabajos como los de Li y Zou (1998) y Forbes (2000), que utilizan muestras de 35 países con medias de cinco años, se argumenta que al utilizar datos en panel desaparece la relación negativa entre crecimiento y desigualdad. Barro (2000) sugiere que el impacto negativo de la desigualdad sobre el crecimiento depende del nivel de riqueza del país, aunque esta relación no sea robusta.

Castelar (2007) hace un análisis de la relación entre crecimiento y desigualdad en los estados brasileños, sobre la base de un panel que abarca el período 1985-2002. Utilizando el método de Arellano y Bond de dos etapas, el autor encuentra que la desigualdad de los ingresos afecta negativamente el crecimiento económico y corrobora la hipótesis de convergencia, de acuerdo con la cual los bajos valores iniciales de los ingresos o del producto conducen a un mayor crecimiento.

También en un análisis relativo al Brasil, Ferreira y Cruz (2010) emplean un modelo con efecto umbral para estudiar la existencia de clubes de convergencia en la desigualdad de los ingresos de los municipios en el período de 1991 a 2000. Los autores encontraron seis clubes de convergencia, donde los factores que redujeron la desigualdad de la distribución de los ingresos en el Brasil actuaron de forma asimétrica en los municipios. En el proceso de convergencia, los ingresos del trabajo fueron más significativos que aquellos provenientes de las transferencias gubernamentales en la reducción de la desigualdad.

\section{Fuente y descripción de los datos}

La base de datos comprende información sobre 25 Unidades de la Federación (UF) para el período de 1981 a $2013^{3}$. Todos los datos se tomaron de la Encuesta Nacional de Hogares (PNAD) realizada por el Instituto Brasileño de Geografía y Estadística (IBGE).

La variable ingresos se refiere a los ingresos familiares per cápita $(R M)$ y se estima a partir de la razón entre el ingreso total de la familia y el número de componentes. A continuación, se determina la media aritmética de esa variable, obteniendo así los ingresos medios para cada una de las UF4.

Las familias que viven con ingresos familiares per cápita insuficientes para satisfacer sus necesidades básicas se clasificaron como pobres. Se utiliza como indicador de pobreza absoluta la medida propuesta por Foster, Greer y Thorbecke (1984), denominada proporción de pobres $\left(P_{0}\right)$, que se define como:

3 Debido a que el período analizado va de 1981 a 2013, los datos relativos al estado de Tocantins, que se creó en 1990, se incorporaron a los del estado de Goiás. Así, a partir de 1990, los cálculos relacionados con los ingresos y los índices de pobreza y desigualdad se realizaron agrupando ambos estados. Asimismo, como la PNAD no se llevó a cabo en los años 1994 y 2000, se optó por hacer una interpolación a partir de la media aritmética entre los años anterior y posterior.

4 Todas las variables monetarias se actualizaron a valores reales de 2013, utilizando como deflactor el índice nacional de precios al consumidor (INPC), con base en octubre de 2013. 


$$
P(\alpha)=\int_{0}^{L P}\left(\frac{L P-y}{L P}\right)^{\alpha} f(y) d y
$$

Donde LP es la línea de pobreza. En el caso de la proporción de pobres $\left(P_{0}\right), \alpha=0$, se adoptó la línea de pobreza utilizada por el Instituto de Investigación Económica Aplicada (IPEA) $)^{5}$, en el caso de la proporción de pobreza extrema, $\alpha=$ ext, con la línea de pobreza extrema $\left(P_{\text {ext }}\right)$ es la parte de la población que se concentra en condiciones de pobreza extrema6 .

Para la desigualdad de los ingresos se utilizó el coeficiente de Gini $(G)^{7}$. Para determinarlo es preciso ordenar en forma ascendente el conjunto de ingresos familiares per cápita para obtener la curva de Lorenz, que relaciona la fracción acumulada de la población con la fracción acumulada de los ingresos en cada percentil. A partir de esta se calcula el coeficiente de Gini para cada UF .

En el cuadro 1 se observa que la proporción de pobres $\left(P_{0}\right)$ media fue de 0,3992 en el período analizado, mientras que los valores mínimo y máximo fueron de 0,0167 y 0,8616, respectivamente. En otras palabras, el 39,92\% de la población brasileña percibía ingresos inferiores a la línea de pobreza adoptada. El 17,76\% de esas personas se encontraba en situación de pobreza extrema.

Los ingresos familiares medios per cápita en el período de 1981 a 2013 fueron de 571,76 reales. La cifra más baja, 160,13 reales, se registró en Piauí en 1983, mientras que la más alta, 1.266,63 reales, se registró en São Paulo en 2012. Por último, la desigualdad media (evaluada por el coeficiente de Gini) fue de 0,5585, variando entre 0,3933 (Roraima en 1983) y 0,6665 (Piauí en 1990).

Cuadro 1

Brasil: estadística descriptiva de las series para todos los estados, 1981-2013

\begin{tabular}{lccccc}
\hline Variables & Observaciones & Media & Desviación estándar & Mínimo & Máximo \\
\hline Proporción de pobres $\left(P_{0}\right)$ & 800 & 0,3992 & 0,1885 & 0,0167 & 0,8616 \\
\hline Pobreza extrema $\left(P_{\text {ext }}\right)$ & 800 & 0,1776 & 0,1309 & 0,0071 & 0,6279 \\
\hline $\begin{array}{l}\text { Ingresos familiares } \\
\text { per cápita }(R m)\end{array}$ & 800 & 571,76 & 225,56 & 160,13 & $1,266,63$ \\
\hline \begin{tabular}{l} 
Coeficiente de Gini $(G)$ \\
\hline
\end{tabular} & 800 & 0,5585 & 0,0440 & 0,3933 & 0,6665 \\
\hline
\end{tabular}

Fuente: Elaboración propia.

\section{Modelo econométrico}

Para cuantificar los efectos del crecimiento económico y de la desigualdad de los ingresos en la pobreza se utiliza un modelo dinámico para datos en panel, descrito por:

$$
\ln \left(P_{k, i t}\right)=\beta_{0}+\beta_{1} \ln \left(P_{k, i t-1}\right)+\beta_{2} \ln \left(R m_{i, t}\right)+\beta_{3} \ln \left(G_{i, t}\right)+v_{t}+u_{i, t}
$$

\footnotetext{
5 La línea de pobreza utilizada es el doble de la línea de pobreza extrema.

6 La línea de pobreza extrema aquí utilizada es una estimación del valor de una canasta de alimentos con el mínimo de calorías necesarias para abastecer adecuadamente a una persona, sobre la base de recomendaciones de la Organización de las Naciones Unidas para la Alimentación y la Agricultura (FAO) y de la Organización Mundial de la Salud (OMS). Esta serie se calculó a partir de las respuestas a la Encuesta Nacional de Hogares (PNAD/IBGE). Véanse más detalles en www. ipeadata.gov.br.

7 De acuerdo con Litchfield (1999), este índice cumple con cuatro de los cinco axiomas de las medidas de desigualdad, a saber: el principio de transferencia de Pigou-Dalton, el principio de independencia de la escala de ingresos, el principio de la población y el principio de simetría. Sin embargo, no cumple con el axioma de descomposición aditiva.

8 El coeficiente de Gini varía de 0 (cero) a 1 (uno). Cuanto más próximo a 1, mayor será la desigualdad de los ingresos y, por el contrario, cuanto más próximo a 0, menor será la desigualdad (Hoffmann, 1998).
} 
Donde $P_{k, i t}$ representa el índice de pobreza $k$, de acuerdo con la línea de pobreza (es decir, $k=0$ para proporción de pobres; y $k=$ ext para la proporción de pobreza extrema); $R m_{i, t}$ corresponde a los ingresos medios per cápita; $G_{i, t}$ es el coeficiente de Gini; $v_{t}$ representa los efectos fijos no observables de los individuos y $u_{i, t}$ es el término de error idiosincrásico. Los subíndices $i$ y $t$ se refieren, respectivamente, a la Unidad de la Federación $i$ y al año.

El modelo está especificado en logaritmos para obtener las elasticidades ingreso y desigualdad de la pobreza, representadas por los coeficientes $\beta_{2}$ y $\beta_{3}$ respectivamente.

La especificación del modelo (2) se basa en la suposición de que la pobreza actual tiende a influir en la dinámica de la pobreza en el próximo período. Esto justifica la presencia de la variable dependiente rezagada un período $\left(P_{k, i t-1}\right)$ como variable explicativa ${ }^{9}$.

Las hipótesis del modelo son que $E\left[v_{i}\right]=E\left[u_{i, t}\right]=E\left[v_{i} u_{i, t}\right]=0$ para $i=1, \ldots, N$ y $t=1, \ldots, T$; el error no está correlacionado temporalmente, o sea, $E\left[u_{i, T} u_{i, S}\right]=0$ para $i=1, \ldots, N$ y $\forall t \neq s$. Además, se impone la condición inicial de que $E\left[P_{k, i t} u_{i, t}\right]=0$ para $i=1, \ldots, N$ y $t=1, \ldots, T$ (Ahn y Schmidt, 1995).

Cabe hacer una observación importante sobre el problema de la endogeneidad. Figueiredo y Laurini (2015) expresan preocupación por la endogeneidad que puede estar presente cuando se utiliza la metodología habitual de Ravallion y Chen (1997) para medir los efectos del crecimiento económico en la pobreza y la desigualdad. Según los autores, esta puede producirse por dos mecanismos: la determinación simultánea de la pobreza y del crecimiento, que generaría una correlación con el componente de error (la endogeneidad puede derivar de factores no observados que afectan a esos dos componentes de manera simultánea o de un proceso de desarrollo financiero que afecta simultáneamente a la pobreza, el crecimiento y la desigualdad), o el efecto directo que una tendencia de crecimiento puede tener en la medida de pobreza.

Otro problema que puede sesgar las estimaciones, también según Figueiredo y Laurini (2015), reside en la forma funcional utilizada para la relación entre pobreza, crecimiento y desigualdad. Con las especificaciones en forma lineal se asume que los efectos del crecimiento en la pobreza y la desigualdad son constantes e independientes de los niveles de crecimiento y desigualdad. Sin embargo, en diversos trabajos de la literatura dedicada al tema se plantean cuestionamientos a ese respecto y se argumenta que la hipótesis de una relación lineal entre crecimiento, desigualdad y pobreza puede sesgar las estimaciones debido a un problema de especificación incorrecta, cuando, de hecho, esas relaciones no serían lineales.

La presencia de $P_{k, i t-1}$ como variable explicativa en la ecuación (2) puede dar lugar al problema de endogeneidad ${ }^{10}$ y producir un sesgo en el panel dinámico. En ese caso, las estimaciones de mínimos cuadrados ordinarios tienden a estar sesgadas y a ser inconsistentes, sobrestimando el coeficiente de la variable mencionada. Es posible tratar de corregir ese sesgo mediante el uso del estimador de efecto fijo dentro de los grupos; ello genera unas desviaciones estándar ligeramente menores de los coeficientes. Sin embargo, para solucionar el problema de endogeneidad, se debe aplicar la transformación del modelo en primera diferencia y proceder a la estimación por el método generalizado de momentos, a partir de la minimización de las condiciones de momentos de la distribución. De este modo, la ecuación (2) se transforma en:

$$
\Delta \ln \left(P_{k, i t}\right)=\beta_{1} \Delta \ln \left(P_{k, i t-1}\right)+\beta_{2} \Delta \ln \left(R m_{i, t}\right)+\beta_{3} \Delta \ln \left(G_{i, t}\right)+\Delta u_{i, t}
$$

\footnotetext{
9 Véase evidencia sobre la perpetuación de la pobreza en el Brasil en Ribas, Machado y Golgher (2006).

10 Caso en que las variables explicativas del modelo se correlacionan con los residuos $E\left(Z_{i}, \varepsilon_{i t}\right) \neq 0$. Una variable cualquiera puede clasificarse como: estrictamente exógena, si no está correlacionada con los términos de error pasados, presente y futuros; débilmente exógena, si está correlacionada solo con valores pasados del término de error; y endógena, si está correlacionada con los términos de error pasados, presentes y futuros.
} 
Donde $\Delta$ es un operador de diferencias y, por la construcción de la ecuación (3), $\Delta \ln \left(P_{k, i t-1}\right)$ y $\Delta u_{i, t}$ están correlacionados y la endogeneidad todavía persiste. De ese modo, se vuelve necesario utilizar algún instrumento para $\Delta \ln \left(P_{k, i t-1}\right)$. El conjunto de hipótesis adoptadas en la ecuación (2) supone que las condiciones de momentos, $E\left[\Delta \ln \left(P_{k, i t-s} \Delta u_{i t}\right)\right]=0$ para $t=3,4, \ldots, T$ y $s \geq 2$ son válidas. De acuerdo con esos momentos, Arellano y Bond (1991) sugieren emplear $\Delta \ln \left(P_{k, i t-s}\right)$, para $t=3,4, \ldots, T$ y $s \geq 2$ como instrumentos para la ecuación (3).

En el segundo caso, los valores de la variable rezagada en uno o más períodos pueden considerarse como instrumentos válidos para estimar la ecuación (3). En el último caso, los valores rezagados en dos o más períodos son instrumentos válidos en la estimación de la ecuación (2).

Sin embargo, Arellano y Bover (1995) y Blundell y Bond (1998) explican que esos instrumentos son débiles cuando las variables, dependientes y explicativas, presentan una marcada persistencia, cuando la varianza relativa de los efectos fijos aumenta o cuando ocurren ambas cosas. En este caso, el estimador es inconsistente y está sesgado. Como solución a este problema, los autores recomiendan la estimación de un sistema que combine el conjunto de ecuaciones en diferencias (ecuación (3)) con el conjunto de ecuaciones en nivel (ecuación (2)), conocido como método MGM para sistemas (System-GMM).

Para las ecuaciones en diferencias, el conjunto de instrumentos es el mismo descrito anteriormente, mientras que para la regresión en nivel, los instrumentos apropiados son las diferencias rezagadas de las respectivas variables. Por ejemplo, asumiendo que las diferencias de las variables explicativas no están correlacionadas con los efectos fijos individuales (para $t=3,4, \ldots, T$ ) y $E\left[\Delta \ln \left(P_{k, i 2} v_{i}\right)\right]=0$ para $i=1,2,3, \ldots, N$, las variables explicativas en diferencias y $\Delta \ln \left(P_{k, i t-1}\right)$, en caso de que estas sean exógenas o débilmente exógenas, son instrumentos válidos para la ecuación en niveles. Lo mismo ocurre si estas son endógenas, pero los instrumentos son las variables explicativas en diferencias rezagadas un período y más $\Delta \ln \left(P_{k, i t-1}\right)$.

La consistencia del estimador System-GMM depende de la ausencia de correlación serial en el término de error y de la validez de los instrumentos adicionales. Inicialmente se prueban las hipótesis nulas de ausencia de autocorrelación de primer y segundo orden de los residuos. Para que los estimadores de los parámetros sean consistentes, la hipótesis de ausencia de autocorrelación de primer orden debe rechazarse y la de segundo orden debe aceptarse. A continuación, se realizan las pruebas de Hansen y de Sargan para verificar la validez de los instrumentos utilizados por el método System-GMM.

Debido a que el estimador de mínimos cuadrados ordinarios para la variable dependiente rezagada teóricamente proporciona estimaciones sesgadas hacia abajo, mientras que los estimadores del método dentro de los grupos generan estimaciones sesgadas hacia arriba, la estimación adecuada

del parámetro de $\ln \left(P_{k, i t-1}\right)$ debe estar comprendida entre los límites de esos dos estimadores (MCO y dentro de los grupos).

Después de esta descripción de los procedimientos econométricos y estadísticos para realizar las estimaciones, en la próxima sección se presentan el análisis y la discusión de los resultados obtenidos con el método System-GMM para que las varianzas de los parámetros sean robustas a la heterocedasticidad y la autocorrelación. El estimador obtenido se corrigió con el método Windmeijer (2005) para evitar la subestimación de las verdaderas varianzas en la muestra finita.

\section{Análisis y discusión de los resultados}

Para comparar las elasticidades ingreso y desigualdad de la pobreza, se optó por estimar el modelo teniendo en cuenta el período de 1981 a 2013 y, además, los subperíodos de 1981 a 1994 y 1995 a 
2013, con el objetivo de analizar las etapas anterior y posterior al Plan Real. De ese modo, se podrán comparar los valores de las elasticidades en distintos períodos de la economía brasileña.

En primer lugar, se estima la ecuación (2) por MCO y por el método dentro de los grupos $\ln \left(R m_{i, t}\right)$. Para seleccionar el modelo estimado, se tienen en cuenta los resultados de las pruebas de especificación de Hausman para verificar si $R m_{i, t}$ y $G_{i, t}$ son endógenas y el análisis de la validez de los instrumentos mediante las pruebas de Hansen y Sargan. De acuerdo con estas pruebas, no es necesario tratar a las variables $R m_{i, t}$ y $G_{i, t}$ como endógenas y, además, la utilización de los segundos rezagos de las variables y los instrumentos adicionales requeridos por el System-GMM son válidos.

Asimismo, se realizaron pruebas para captar la presencia de autocorrelación de primer orden de los residuos en nivel y en primera diferencia (es decir, segundo orden), cuyos resultados se muestran en los cuadros 2 a 4 . Se observa que en ninguna de las estimaciones se rechaza la hipótesis nula, es decir, que los residuos presentan una correlación de primer orden. Para que las estimaciones no estén sesgadas, es necesario mitigar su presencia. Uno de los mecanismos para ello consiste en estimar el modelo en primera diferencia, por ejemplo mediante el estimador System-GMM.

En consecuencia, a los efectos del análisis de los resultados se tienen en cuenta las estimaciones obtenidas mediante el System-GMM. En los cuadros 2 a 4 se presentan los resultados de los modelos seleccionados y estimados por MCO y por el método dentro de los grupos para la proporción de pobres $\left(P_{0}\right)$ y la proporción de pobreza extrema $\left(P_{\text {ext }}\right)$ en el Brasil. Las estimaciones por MCO y dentro de los grupos se presentan solo para mostrar que el valor estimado de la variable dependiente rezagada se encuentra entre los valores estimados obtenidos mediante esos dos métodos. Estas funcionan como un posible indicador de que el sesgo causado por la presencia de variables endógenas y de efectos fijos no observables se corrige con el método System-GMM.

\section{Período de 1981 a 2013}

En el cuadro 2 se presentan los resultados de las elasticidades ingreso y desigualdad de la proporción de pobres y de pobreza extrema relativos al período de 1981 a 2013. La hipótesis de persistencia de la pobreza parece confirmarse, pues se verifica la significación estadística de la variable $\ln \left(P_{k, i t-1}\right)$. En ese sentido, se verifica que la persistencia está presente con respecto a la proporción de pobres ${ }^{11}$ $\left(0,1025\right.$ para $\left.P_{0}\right)$ y es mayor en el caso de la pobreza extrema $\left(0,1356\right.$ para $\left.P_{\text {ext }}\right)$. Estos resultados indican que, aunque no es explosiva, la pobreza en los estados brasileños es persistente ${ }^{12}$, y corroboran los estudios realizados por Ribas, Machado y Golgher (2006), Marinho y Araújo (2010) y Marinho, Linhares y Campelo (2011).

Entre los factores determinantes considerados que afectan la pobreza, los ingresos familiares medios per cápita y el coeficiente de Gini contribuyeron significativamente a su reducción.

Los coeficientes estimados de las elasticidades ingreso de la pobreza presentaron los signos esperados, en este caso negativos, pues el aumento de los ingresos familiares medios per cápita reduce los niveles de pobreza. El valor de los coeficientes fue de -0,9235 para $P_{0}$ y $-1,1418$ para $P_{\text {ext }}$. Eso significa que un aumento del 10\% en los ingresos familiares medios per cápita en el Brasil se traduce en una reducción del 9,23\% en la proporción de pobres y en una disminución del 11,41\% en la proporción de pobreza extrema.

Con respecto al crecimiento a favor de los pobres, se observa que, según la corriente de Kakwani y Pernia (2000), los valores de las elasticidades ingreso de la pobreza indicarían que este

\footnotetext{
${ }^{11}$ Estadísticamente significativo al $1 \%$.

12 De acuerdo con Rocha (2006), el nivel de pobreza en el Brasil es elevado y se relaciona, sobre todo, con la mala distribución de los recursos entre sus habitantes.
} 
se verifica solo en el caso de la pobreza extrema, pues el coeficiente estimado es superior a 1 (en términos absolutos). Eso significa que la reducción de los niveles de pobreza extrema es superior al crecimiento de los niveles de ingresos. Sin embargo, de acuerdo con la corriente de Ravallion y Chen (2003), hay crecimiento a favor de los pobres tanto en el caso de la pobreza como de la pobreza extrema, pues el crecimiento redujo la pobreza.

En el caso de la elasticidad desigualdad de la pobreza, los coeficientes estimados presentan los signos esperados, es decir positivos, pues el aumento del índice de desigualdad (coeficiente de Gini) incrementa los niveles de pobreza. El valor de los coeficientes fue de 1,9470 para $P_{0}$ y 2,8912 para $P_{\text {ext }}$. En otras palabras, una disminución del $10 \%$ en la desigualdad de los ingresos se traduce en una reducción del 19,47\% en la proporción de pobres y del 28,91\% en la proporción de pobreza extrema en el Brasil.

\section{Cuadro 2}

Brasil: resultados de la estimación para la proporción de pobres y de pobreza extrema, 1981-2013

\begin{tabular}{|c|c|c|c|c|c|c|}
\hline \multirow[b]{2}{*}{ Variables explicativas } & \multicolumn{3}{|c|}{ Pobreza (LnPo) } & \multicolumn{3}{|c|}{ Pobreza extrema $\left(\mathrm{LnP}_{\mathrm{ext}}\right)$} \\
\hline & $\begin{array}{l}\text { Mínimos } \\
\text { cuadrados } \\
\text { ordinarios }\end{array}$ & $\begin{array}{l}\text { Dentro de } \\
\text { los grupos }\end{array}$ & GMM-System & $\begin{array}{c}\text { Mínimos } \\
\text { cuadrados } \\
\text { ordinarios }\end{array}$ & $\begin{array}{l}\text { Dentro de } \\
\text { los grupos }\end{array}$ & GMM-System \\
\hline $\ln P_{0, i t-1}$ & $\begin{array}{c}0,2001^{*} \\
(0,0255)\end{array}$ & $\begin{array}{c}0,0862^{*} \\
(0,0247)\end{array}$ & $\begin{array}{r}0,1025^{\star} \\
(0,0385)\end{array}$ & $\begin{array}{l}0,3596^{*} \\
(0,0231)\end{array}$ & $\begin{array}{l}0,1254 * \\
(0,0208)\end{array}$ & $\begin{array}{r}0,1356^{*} \\
(0,0252)\end{array}$ \\
\hline $\ln R m_{i t}$ & $\begin{array}{l}-0,6732^{*} \\
(0,0311)\end{array}$ & $\begin{array}{l}-0,9476^{\star} \\
(0,0325)\end{array}$ & $\begin{array}{l}-0,9235^{\star} \\
(0,1130)\end{array}$ & $\begin{array}{c}-1,0271^{*} \\
(0,0418)\end{array}$ & $\begin{array}{c}-1,3882^{*} \\
(0,0388)\end{array}$ & $\begin{array}{r}-1,1418^{*} \\
(0,0705) \\
\end{array}$ \\
\hline $\ln G_{i t}$ & $\begin{array}{l}1,3401^{*} \\
(0,1065)\end{array}$ & $\begin{array}{l}2,0793^{*} \\
(0,1069)\end{array}$ & $\begin{array}{l}1,9470^{*} \\
(0,2364)\end{array}$ & $\begin{array}{l}2,1421^{*} \\
(0,1334)\end{array}$ & $\begin{array}{l}3,2094^{*} \\
(0,1224)\end{array}$ & $\begin{array}{l}2,8912^{*} \\
(0,2352)\end{array}$ \\
\hline Constante & $\begin{array}{c}4,3633^{*} \\
(0,1895)\end{array}$ & $\begin{array}{c}6,3059^{*} \\
(0,1985)\end{array}$ & $\begin{array}{r}6,0746^{\star} \\
(0,6982)\end{array}$ & $\begin{array}{c}6,3827^{*} \\
(0,2426)\end{array}$ & $\begin{array}{c}8,8043^{*} \\
(0,2282)\end{array}$ & $\begin{array}{c}8,3485^{\star} \\
(0,4189)\end{array}$ \\
\hline Estadístico F & 2262,58 & 1382,37 & 127,22 & 3156,57 & 2055,37 & 410,18 \\
\hline Valor $p$ & 0,0000 & 0,0000 & 0,0000 & 0,0000 & 0,0000 & 0,0000 \\
\hline $\mathrm{R}^{2}$ & 0,8975 & - & - & 0,9306 & - & - \\
\hline N & 775 & 775 & 775 & 775 & 775 & 775 \\
\hline Grupos & 25 & 25 & 25 & 25 & 25 & 25 \\
\hline \multicolumn{2}{|c|}{$\begin{array}{l}\mathrm{H}_{0} \text { : Ausencia de autocorrelación en } \\
\text { los residuos de primer orden }\end{array}$} & Valor $p$ & 0,012 & & 0,026 & \\
\hline \multicolumn{2}{|c|}{$\begin{array}{l}\mathrm{H}_{0} \text { : Ausencia de autocorrelación en } \\
\text { los residuos de segundo orden }\end{array}$} & Valor $p$ & 0,241 & & 0,921 & \\
\hline \multicolumn{2}{|l|}{ Prueba de Hansen } & Prob>chi2 & 0,450 & & 0,367 & \\
\hline \multicolumn{2}{|l|}{ Prueba de Sargan } & Prob>chi2 & 0,637 & & 0,621 & \\
\hline
\end{tabular}

Fuente: Elaboración propia.

Nota: * denota la significación estadística al nivel del $1 \%$.

Otro resultado importante es que las repercusiones del efecto ingreso y del efecto desigualdad son mayores con respecto a la proporción de pobreza extrema que a la proporción de pobres $\left(P_{0}\right)$. Esto se debe a que la primera se basa en una línea de pobreza inferior, que la vuelve más sensible a las oscilaciones de aumento de los ingresos y reducción de los niveles de desigualdad.

Se observa también que el valor del coeficiente de elasticidad desigualdad de la pobreza es mayor (en términos absolutos) que el de elasticidad ingreso de la pobreza, tanto con respecto a la proporción de pobres como a la proporción de pobreza extrema. Esos resultados indican la posibilidad de que los efectos de la reducción de la desigualdad en la disminución de los niveles de pobreza sean mayores que los del mero incremento de los ingresos medios. Una posible explicación es que los 
aumentos de ingresos se transmiten de manera desproporcional (o desigual) a la población pobre de la región. Estos resultados son similares a los obtenidos por França (2010).

En este sentido, de acuerdo con Ravallion (1997 y 2004), Marinho y Soares (2003), Bourguignon (2002), López y Seven (2004), Menezes y Pinto (2005), Barreto, França y De Oliveira (2008) y Marinho, Linhares y Campelo (2011), las políticas de combate a la pobreza a través del crecimiento serían más eficaces si estuvieran acompañadas por medidas de redistribución de los ingresos.

Como se indicó en la Introducción, el principal aporte de este trabajo consiste en analizar los efectos del crecimiento económico y de la desigualdad de los ingresos en la pobreza del Brasil teniendo en cuenta diferentes períodos de la economía brasileña: un período anterior a la implementación del Plan Real, cuando la inflación representaba un problema endémico en el país, y un período posterior al Plan Real, caracterizado por la estabilización de los precios. Dicho análisis se presenta a continuación.

\section{Período anterior al Plan Real (1981-1994)}

En el cuadro 3 se detallan los resultados de los modelos seleccionados y estimados por MCO, el método entre grupos y System-GMM para la proporción de pobres $\left(P_{0}\right)$ y la proporción de pobreza extrema $\left(P_{\text {ext }}\right)$, considerando el período 1981-1994, anterior a la puesta en marcha del Plan Real.

Una vez más, la hipótesis de persistencia de la pobreza parece confirmarse cuando se verifica la significación estadística de la variable $\ln \left(P_{k, i t-1}\right)$. Se observa que la persistencia es intensa con respecto a la proporción de pobres $\left(0,1212\right.$ para $\left.P_{0}\right)$, y menor que la correspondiente al período 1981-2013 en el caso de la pobreza extrema $\left(0,1230\right.$ para $\left.P_{\text {ext }}\right)$. Esos resultados indican que, aunque no es explosiva, la pobreza en los estados brasileños es persistente.

Cuadro 3

Brasil: resultados de la estimación para la proporción de pobres y de pobreza extrema, 1981-1994

\begin{tabular}{|c|c|c|c|c|c|c|}
\hline \multirow[b]{2}{*}{ Variables explicativas } & \multicolumn{3}{|c|}{ Pobreza (LnPo) } & \multicolumn{3}{|c|}{ Pobreza extrema ( $\left(\mathrm{LnP}_{\text {ext }}\right)$} \\
\hline & $\begin{array}{c}\text { Mínimos } \\
\text { cuadrados } \\
\text { ordinarios }\end{array}$ & $\begin{array}{l}\text { Dentro de } \\
\text { los grupos }\end{array}$ & GMM-System & $\begin{array}{l}\text { Mínimos } \\
\text { cuadrados } \\
\text { ordinarios }\end{array}$ & $\begin{array}{l}\text { Dentro de } \\
\text { los grupos }\end{array}$ & GMM-System \\
\hline $\ln P_{0, i t-1}$ & $\begin{array}{r}0,0353^{*} \\
(0,0168)\end{array}$ & $\begin{array}{c}0,1493^{*} \\
(0,0211)\end{array}$ & $\begin{array}{l}0,1212^{*} \\
(0,0264)\end{array}$ & $\begin{array}{l}0,1607^{*} \\
(0,0330)\end{array}$ & $\begin{array}{l}0,0495^{\star \star \star} \\
(0,0282)\end{array}$ & $\begin{array}{r}0,1230^{*} \\
(0,0421)\end{array}$ \\
\hline $\ln R m_{i t}$ & $\begin{array}{l}-0,9131^{*} \\
(0,0411)\end{array}$ & $\begin{array}{l}-1,1542^{*} \\
(0,0511)\end{array}$ & $\begin{array}{l}-0,7813^{\star} \\
(0,0705)\end{array}$ & $\begin{array}{l}-1,2910^{\star} \\
(0,0581)\end{array}$ & $\begin{array}{l}-1,7918^{*} \\
(0,0630)\end{array}$ & $\begin{array}{r}-0,8607^{*} \\
(0,0791)\end{array}$ \\
\hline $\ln G_{i t}$ & $\begin{array}{r}1,5575^{*} \\
(0,1483)\end{array}$ & $\begin{array}{l}2,2704^{*} \\
(0,1535)\end{array}$ & $\begin{array}{l}1,7487^{*} \\
(0,3864)\end{array}$ & $\begin{array}{l}2,1949^{\star} \\
(0,1912)\end{array}$ & $\begin{array}{l}3,4138^{*} \\
(0,1883)\end{array}$ & $\begin{array}{l}2,6541^{*} \\
(0,2532)\end{array}$ \\
\hline Constante & $\begin{array}{l}5,6512^{*} \\
(0,2458)\end{array}$ & $\begin{array}{r}8,6960^{*} \\
(0,3363)\end{array}$ & $\begin{array}{r}6,2928^{*} \\
(0,5381)\end{array}$ & $\begin{array}{l}7,6854^{*} \\
(0,3349)\end{array}$ & $\begin{array}{r}11,2595^{*} \\
(0,4100)\end{array}$ & $\begin{array}{l}8,8164^{*} \\
(0,4758)\end{array}$ \\
\hline Estadístico F & 626,03 & 281,22 & 83,17 & 991,05 & 374,07 & 180,89 \\
\hline Valor $p$ & 0,0000 & 0,0000 & 0,0000 & 0,0000 & 0,0000 & 0,0000 \\
\hline$R^{2}$ & 0,8543 & - & - & 0,9051 & - & - \\
\hline N & 325 & 325 & 325 & 325 & 325 & 325 \\
\hline Grupos & 25 & 25 & 25 & 25 & 25 & 25 \\
\hline \multicolumn{2}{|c|}{$\begin{array}{l}\mathrm{H}_{0} \text { : Ausencia de autocorrelación en } \\
\text { los residuos de primer orden }\end{array}$} & Valor $p$ & 0,040 & & 0,013 & \\
\hline \multicolumn{2}{|c|}{$\begin{array}{l}H_{0} \text { : Ausencia de autocorrelación en } \\
\text { los residuos de segundo orden }\end{array}$} & Valor $p$ & 0,134 & & 0,892 & \\
\hline \multicolumn{2}{|l|}{ Prueba de Hansen } & Prob>chi2 & 0,369 & & 0,425 & \\
\hline \multicolumn{2}{|l|}{ Prueba de Sargan } & Prob>chi2 & 0,425 & & 0,538 & \\
\hline
\end{tabular}

Fuente: Elaboración propia.

Nota: $\quad{ }^{*} \mathrm{y}^{* \star *}$ denotan la significación estadística al nivel del $1 \%$ y el $10 \%$, respectivamente. 
Al igual que en el análisis del período completo, en esta submuestra se concluye que los ingresos familiares medios per cápita y el coeficiente de Gini, considerados aquí como los determinantes de la pobreza, realmente contribuyeron de forma significativa a su reducción.

De la misma forma, como se anticipó en el análisis anterior, los coeficientes de las elasticidades ingreso de la pobreza presentan signos negativos, que es lo que se esperaba, pues el aumento de los ingresos familiares medios per cápita reduce los niveles de pobreza. El valor de los coeficientes fue de -0,7813 para $P_{0}$ y -0,8607 para $P_{\text {ext }}$. La interpretación de esas cifras indica que un aumento del $10 \%$ en los ingresos familiares medios per cápita en el Brasil se traduce en una reducción del 7,81\% en la proporción de pobres y en una disminución del 8,61\% en la proporción de pobreza extrema. Si bien no habría crecimiento a favor de los pobres conforme la corriente de Kakwani y Pernia (2000), este se verificaría según la corriente de Ravallion y Chen (2003), pues el crecimiento reduce la pobreza.

Los coeficientes estimados para la elasticidad desigualdad de la pobreza presentan nuevamente signos positivos, pues el aumento del índice de desigualdad (coeficiente de Gini) eleva los niveles de pobreza. El valor de los coeficientes fue de 1,7487 para $P_{0}$ y 2,6541 para $P_{\text {ext }}$. En otras palabras, una disminución del 10\% en la desigualdad de los ingresos se traduce en una reducción del 17,49\% en la proporción de pobres y del 26,54\% en la proporción de pobreza extrema en el Brasil.

Al igual que antes, las repercusiones del efecto ingreso y del efecto desigualdad son mayores con respecto a la proporción de pobreza extrema que a la proporción de pobres $\left(P_{0}\right)$.

\section{Período posterior al Plan Real (1995-2013)}

En el cuadro 4 se resumen los resultados de las mismas estimaciones presentadas en los apartados anteriores, empleando las metodologías ya señaladas, para el período posterior al Plan Real.

La hipótesis de persistencia de la pobreza se confirma una vez más, al verificarse la significación estadística de la variable $\ln \left(P_{k, i t-1}\right)$. La persistencia es nuevamente intensa con respecto a la proporción de pobres $\left(0,7690\right.$ para $\left.P_{0}\right)$ y menor en el caso de la pobreza extrema (0,5905 para $\left.P_{\text {ext }}\right)$. Esos resultados, como los del análisis del período completo y de la submuestra del período anterior al Plan Real, indican que la pobreza en los estados brasileños es persistente, aunque no explosiva.

En forma análoga, los ingresos familiares medios per cápita y el coeficiente de Gini también contribuyeron significativamente a la reducción de la pobreza en el período posterior al Plan Real.

Los coeficientes estimados de elasticidad ingreso de la pobreza presentan el mismo signo negativo, corroborando que el aumento de los ingresos familiares medios per cápita reduce los niveles de pobreza. El valor de los coeficientes fue de $-1,1560$ para $P_{0}$ y $-1,3410$ para $P_{\text {ext }}$. En otras palabras, un aumento del $10 \%$ en los ingresos familiares medios per cápita en el Brasil se traduce en una reducción del 11,56\% en la proporción de pobres y en una disminución del 13,41\% en la proporción de pobreza extrema. En consecuencia, como se explicó anteriormente, se observa un crecimiento a favor de los pobres con respecto a la pobreza y a la pobreza extrema, tanto para la corriente de Kakwani y Pernia (2000) como para la de Ravallion y Chen (2003).

Los coeficientes de elasticidad desigualdad de la pobreza estimados presentan el signo positivo esperado, que significa que la disminución del índice de Gini reduce los niveles de pobreza. El valor de los coeficientes fue de 2,0564 para $P_{0}$ y 2,2508 para $P_{\text {ext }}$. En otras palabras, una reducción del 10\% en la desigualdad de los ingresos se traduce en una reducción del 20,56\% en la proporción de pobres y del $22,50 \%$ en la proporción de pobreza extrema en el Brasil.

Al igual que en todo el período analizado y en el período de 1981 a 1994, las repercusiones del efecto ingreso y del efecto desigualdad son mayores con respecto a la proporción de pobreza extrema que a la proporción de pobres $\left(P_{0}\right)$. 
La explicación, como ya se mencionó, es que la primera se basa en una línea de pobreza inferior, que la vuelve más sensible a las oscilaciones de aumento de los ingresos. En resumen, todos los resultados correspondientes a los diferentes subperíodos (el período anterior al Plan Real, caracterizado por una inflación considerable y descontrolada, y el período posterior al Plan Real, con estabilidad y bajos niveles de inflación) son similares y concuerdan con los encontrados con respecto al período completo de la muestra. No obstante, las elasticidades ingreso y desigualdad de la pobreza, tanto con respecto a la proporción de pobres como a la pobreza extrema, son menores (en valores absolutos) en el período anterior a la implementación del Plan Real, que en los otros dos períodos analizados.

Cuadro 4

Brasil: resultados de la estimación para la proporción de pobres y de pobreza extrema, 1981-2012

\begin{tabular}{|c|c|c|c|c|c|c|}
\hline \multirow[b]{2}{*}{ Variables explicativas } & \multicolumn{3}{|c|}{ Pobreza (LnPo) } & \multicolumn{3}{|c|}{ Pobreza extrema (LnPext) } \\
\hline & $\begin{array}{l}\text { Mínimos } \\
\text { cuadrados } \\
\text { ordinarios }\end{array}$ & $\begin{array}{l}\text { Dentro de } \\
\text { los grupos }\end{array}$ & GMM-System & $\begin{array}{l}\text { Mínimos } \\
\text { cuadrados } \\
\text { ordinarios }\end{array}$ & $\begin{array}{l}\text { Dentro de } \\
\text { los grupos }\end{array}$ & GMM-System \\
\hline $\ln P_{0, i t-1}$ & $\begin{array}{r}0,8834^{*} \\
(0,0243) \\
\end{array}$ & $\begin{array}{c}0,6641^{*} \\
(0,0306)\end{array}$ & $\begin{array}{r}0,7690^{*} \\
(0,0656) \\
\end{array}$ & $\begin{array}{r}0,6948^{*} \\
(0,0306)\end{array}$ & $\begin{array}{c}0,3068^{*} \\
(0,0356)\end{array}$ & $\begin{array}{r}0,5905^{*} \\
(0,0613)\end{array}$ \\
\hline $\ln R m_{i t}$ & $\begin{array}{l}-0,7596^{*} \\
(0,0333)\end{array}$ & $\begin{array}{l}-0,8641^{*} \\
(0,0398)\end{array}$ & $\begin{array}{r}-1,1560^{*} \\
(0,0739)\end{array}$ & $\begin{array}{l}-0,9371^{*} \\
(0,0578)\end{array}$ & $\begin{array}{l}-1,0503^{*} \\
(0,0651)\end{array}$ & $\begin{array}{r}-1,3410^{*} \\
(0,1044)\end{array}$ \\
\hline $\ln G_{i t}$ & $\begin{array}{r}0,6444^{*} \\
(0,0971)\end{array}$ & $\begin{array}{l}1,3040^{*} \\
(0,1149)\end{array}$ & $\begin{array}{r}2,0564^{*} \\
(0,3547) \\
\end{array}$ & $\begin{array}{c}1,5687^{*} \\
(0,1686)\end{array}$ & $\begin{array}{l}2,9973^{*} \\
(0,1797)\end{array}$ & $\begin{array}{l}2,2508^{*} \\
(0,2387)\end{array}$ \\
\hline Constante & $\begin{array}{c}1,2597^{*} \\
(0,2014)\end{array}$ & $\begin{array}{l}2,9136^{*} \\
(0,2360)\end{array}$ & $\begin{array}{l}2,0740^{*} \\
(0,4853)\end{array}$ & $\begin{array}{l}3,2547^{*} \\
(0,3391)\end{array}$ & $\begin{array}{l}6,2843^{*} \\
(0,2739)\end{array}$ & $\begin{array}{l}4,2841^{*} \\
(0,6008)\end{array}$ \\
\hline Estadístico F & 4664,63 & 1781,79 & 1090,76 & 3025,40 & 1319,33 & 1050,62 \\
\hline Valor $p$ & 0,0000 & 0,0000 & 0,0000 & 0,0000 & 0,0000 & 0,0000 \\
\hline $\mathrm{R}^{2}$ & 0,9708 & - & - & 0,9557 & - & - \\
\hline $\mathrm{N}$ & 425 & 425 & 425 & 425 & 425 & 425 \\
\hline Grupos & 25 & 25 & 25 & 25 & 25 & 25 \\
\hline $\begin{array}{l}\mathrm{H}_{0} \text { : Ausencia de autocorrelación en } \\
\text { los residuos de primer orden }\end{array}$ & & Valor $p$ & 0,008 & & 0,011 & \\
\hline $\begin{array}{l}\mathrm{H}_{0} \text { : Ausencia de autocorrelación en } \\
\text { los residuos de segundo orden }\end{array}$ & & Valor $p$ & 0,805 & & 0,496 & \\
\hline Prueba de Hansen & & Prob >chi2 & 0,762 & & 0,453 & \\
\hline Prueba de Sargan & & Prob>chi2 & 0,835 & & 0,792 & \\
\hline
\end{tabular}

Fuente: Elaboración propia.

Nota: * denota la significación estadística al nivel del $1 \%$.

Esto probablemente deriva de la inestabilidad observada en la economía brasileña durante la década de 1980, cuando se registraron elevadas tasas de inflación combinadas con un bajo crecimiento económico. Se puede decir que eso determina una menor sensibilidad de los niveles de pobreza con respecto a las oscilaciones de los ingresos y la desigualdad.

Como se suponía, los resultados indican también la posibilidad de que los efectos de la reducción de la desigualdad en la disminución de los niveles de pobreza sean mayores que los del mero incremento de los ingresos medios. Se reitera que una posible explicación para ese fenómeno es que los aumentos de los ingresos se transmiten de manera desproporcional o desigual a la población pobre de la región, es decir, que la política de transferencia de los ingresos no se concentra en las personas más desfavorecidas. 


\section{Consideraciones finales}

El objetivo de este artículo era analizar los efectos del crecimiento económico y la desigualdad de los ingresos en la pobreza del Brasil entre 1981 y 2013, empleando una metodología de datos en panel dinámico y dos medidas de pobreza, a saber: la proporción de pobres y la pobreza extrema. Se estudiaron tres períodos: el primero va de 1981 a 2013, mientras que el segundo y el tercero corresponden a las etapas anterior y posterior a la implementación del Plan Real (1981-1994 y 19952013, respectivamente).

A partir de los resultados estimados por los métodos de MCO, dentro de los grupos y SystemGMM, se puede afirmar que existe persistencia en la dinámica de la pobreza y que esta puede relacionarse, sobre todo, con la mala distribución de los ingresos en la sociedad brasileña.

Al analizar el período anterior al Plan Real (1981-1994), se observa que las elasticidades ingreso y desigualdad de la pobreza, tanto con respecto a la proporción de pobres como a la pobreza extrema, son menores (en valores absolutos) que en los otros dos períodos analizados. Eso probablemente deriva de la inestabilidad observada en la economía brasileña durante la década de 1980, cuando se registraron elevadas tasas de inflación combinadas con un bajo incremento de los ingresos. Se puede decir que eso determinó una menor sensibilidad de los niveles de pobreza a las oscilaciones de los ingresos y la desigualdad.

El análisis de la elasticidad ingreso de la pobreza indica un crecimiento a favor de los pobres en el período 1981-2013 según la corriente de Kakwani y Pernia (2000), pero solo en el caso de la pobreza extrema. Eso significa que el aumento de los ingresos fue superior a la reducción de la pobreza. El crecimiento a favor de los pobres según la definición de Ravallion y Chen (2003) se observó en todos los períodos analizados, tanto en el caso de la proporción de pobres como en el de la proporción de pobreza extrema.

Si bien en el subperíodo anterior al Plan Real no se observó crecimiento a favor de los pobres en relación con ninguna corriente, este sí se verificó en el subperíodo posterior, tanto en relación con los niveles de pobreza como con respecto a la pobreza extrema. Ese resultado puede explicarse en virtud de la estabilidad económica que caracterizó al período en cuestión, el aumento real del salario mínimo y las políticas de transferencia directa de ingresos (França, 2010; Kakwani, Neri y Son, 2010).

A partir de las elasticidades se verificó que tanto las políticas destinadas a aumentar los ingresos familiares medios per cápita como las que procuran la reducción de la desigualdad tienden a reducir los dos niveles de pobreza examinados. Asimismo, se puede decir que estas son más eficientes para reducir la proporción de pobreza extrema que la proporción de pobres, debido a que la primera se basa en una línea de pobreza inferior a la segunda y, por consiguiente, presenta una mayor sensibilidad a las políticas adoptadas.

También en ese sentido, los efectos de la reducción de la desigualdad en la disminución de los dos niveles de pobreza (proporción de pobres y proporción de indigentes) son mayores que los de las políticas únicamente orientadas al crecimiento de los ingresos medios. O sea, de acuerdo con los resultados presentados, las políticas de crecimiento económico que promueven simultáneamente el aumento de los ingresos y la reducción de las disparidades (por ejemplo, las políticas concentradas en los más pobres como el programa Bolsa Familia) tienden a reducir más la pobreza en el Brasil que las políticas de crecimiento económico cuyo único objetivo es el aumento de los ingresos medios. 


\section{Bibliografía}

Adams, R.H. (2004), "Economic growth, inequality and poverty: estimating the growth elasticity of poverty", World Development, vol. 32, № 12, Amsterdam, Elsevier.

Ahn, S.C. y P. Schmidt (1995), "Efficient estimation of models for dynamic panel data", Journal of Econometrics, vol. 68, No 1, Amsterdam, Elsevier.

Araújo, J.A., F.J.S. Tabosa y A.S. Khan (2012), "Elasticidade renda e elasticidade desigualdade da pobreza no Nordeste brasileiro", Revista de Política Agrícola, № 1.

Arellano, M. y S. Bond (1991), "Some tests of specification for panel data: Monte Carlo evidence and an application to employment equations", The Review of Economic Studies, vol. 58, № 2, Oxford University Press.

Arellano, M. y O. Bover (1995), "Another look at the instrumental-variable estimation of error components model", Journal of Econometrics, vol. 68, № 1, Amsterdam, Elsevier.

Banerjee, A. y E. Duflo (2003), "Inequality and growth: what can the data say?", Journal of Economic Growth, vol. $8, N^{\circ} 3$, Springer.

Barreto, F. (2005), "Crescimento econômico, pobreza e desigualdade: o que sabemos sobre eles?", Série Ensaios Sobre Pobreza, № 1, Fortaleza.

Barreto, F., J.M. França y V.H. de Oliveira (2008), "O que mais importa no combate à pobreza, crescimento econômico ou redução da desigualdade: evidências para as regiões brasileiras”, Série Ensaios Sobre Pobreza, № 16, Fortaleza.

Barreto, F., P.J. Melo Neto y E. Tebaldi (2001), "Desigualdade de renda e crescimento econômico no nordeste brasileiro", Revista Econômica do Nordeste, vol. 32, № 9.

Barro, R. (2000), "Inequality and growth in a panel of countries", Journal of Economic Growth, vol. 5, № 1, Springer.

Barros, P.R. y otros (2007), "Determinantes imediatos da queda da desigualdade de renda brasileira", Texto para Discussão, № 1253, Río de Janeiro, Instituto de Investigación Económica Aplicada (IPEA).

Blundell, R. y S. Bond (1998), "Initial conditions and moment restrictions in dynamic panel data models", Journal of Econometrics, vol. 87, № 1, Amsterdam, Elsevier.

Bourguignon, F. (2002), "The growth elasticity of poverty reduction: explaining heterogeneity across countries and time periods", Inequality and Growth: Theory and Policy Implications, T. Eicher y S. Turnovsky, Cambridge, Massachusetts, The MIT Press.

Castelar, P. (2007), "Crescimento econômico e desigualdade de renda no Brasil: uma análise de painel dinâmico para o período 1985-2002", Fortaleza, Universidad Federal de Ceará.

Chen, S. e Y. Wang (2001), "China's growth and poverty reduction: recent trends between 1990 and 1999", Policy Research Working Paper, № 2651, Washington, D.C., Banco Mundial.

De Lima, F.S., F. Barreto y E. Marinho (2003), "Impacto do crescimento econômico e da concentração de renda sobre o nível de pobreza dos estados brasileiros", Anais do VII Encontro Regional de Economia, Fortaleza.

Deineger, K. y L. Squire (1998), "New ways of looking at old issues: inequality and growth", Journal of Economic Development, vol. 57, № 2, Amsterdam, Elsevier.

Diniz, M.B. (2005), "Contribuições ao estudo da desigualdade de renda entre os estados brasileiros", tesis, Fortaleza, Universidad Federal de Ceará.

Easterly, W. (2000), "The Effect of IMF and World Bank Programs on Poverty", Washington, D.C., Banco Mundial, inédito.

Ferreira, F. (1999), A Brief Overview of Theories of Growth and Distribution, Washington, D.C., Banco Mundial.

Ferreira, F., P. Lanjouw y M.C. Neri (2003), "A robust poverty profile for Brazil using multiple data sources", Revista Brasileira de Economia, vol. 57, № 1.

Ferreira, R.T. y M.S. Cruz (2010), "Efeitos da educação, da renda do trabalho, das transferências e das condições iniciais na evolução da desigualdade de renda nos municípios brasileiros no período de 1991 a 2000", Pesquisa e Planejamento Econômico, vol. 40, № 1.

Fields, G. y G. Jakubson (1994), "The Inequality-Development Relationship in Developing Countries", inédito.

Figueiredo, E. y M.P. Laurini (2015), "Poverty elasticity: a note on a new empirical approach", Review of Income and Wealth, vol. 62, № 2, Wiley.

Fishlow, A. (1972), "Brazilian size distribution of income", American Economic Review, vol. 62, № 2, Nashville, Tennessee, American Economic Association. 
Forbes, K.A. (2000), "A reassessment of the relationship between inequality and growth", American Economic Review, vol. 90, № 4, Nashville, Tennessee, American Economic Association.

Foster, J., J. Greer y E. Thorbecke (1984), "A class of decomposable poverty measures", Econometrica, vol. 52, N 3, Nueva York, The Econometric Society.

Fosu, A.K. (2010), "Growth, inequality, and poverty reduction in developing countries: recent global evidence", Working Paper, № 2011/01, Instituto Mundial de Investigaciones de Economía del Desarrollo (UNU-WIDER).

França, J.M.S. (2010), "Crescimento pró-pobre no Brasil: impactos regionais", tesis, Río de Janeiro.

Hoffmann, R. (2005), "Elasticidade da pobreza em relação à renda média e à desigualdade no Brasil e nas unidades da federação", Economia, vol. 6, № 2 .

(1998), Distribuição de renda: medidas de desigualdade de renda e pobreza, São Paulo, Editora da Universidade de São Paulo (EDUSP).

IPEA (Instituto de Investigación Económica Aplicada), "IPEADATA" [en línea] www.ipeadata.gov.br.

$\mathrm{Li}, \mathrm{H}$. y H. Zou (1998), "Income inequality is not harmful for growth: theory and evidence", Review of Development Economics, vol. 2, No 3, Wiley Blackwell.

Litchfield, J.A. (1999), "Inequality: methods and tools", inédito.

López, J.H. y L. Seven (2004), "The mechanics of growth-poverty-inequality relationship", inédito.

Kakwani, K., S. Khandker y H. Son (2004), "Pro-poor growth: concepts and measurement with country case studies", Working Paper, N 1, Brasilia, Centro Internacional de la Pobreza.

Kakwani, N., M.C. Neri y H. Son (2010), "Linkages between pro-poor growth, social programs and labor market: the recent Brazilian experience", World Development, vol. 38, Nㅜ 6, Amsterdam, Elsevier.

Kakwani, K. y E. Pernia (2000), "What is pro-poor growth?", Asian Development Review, vol. 18, № 1, Banco Asiático de Desarrollo.

Kraay, A. (2004), "When is growth pro-poor? Cross-country evidence", Policy Research Working Paper Series, $N^{\circ} 3225$, Washington, D.C., Banco Mundial.

Kuznets, S. (1955), "Economic growth and income inequality", American Economic Review, vol. 45, Nashville, Tennessee, American Economic Association.

Manso, C.A., F. Barreto y E. Tebaldi (2006), "O desequilíbrio regional brasileiro: novas perspectivas a partir das fontes de crescimento pró-pobre", Série Ensaios sobre Pobreza, Nㅜ 6, Fortaleza.

Marinho, E. y F. Soares (2003), "Impacto do crescimento econômico e da concentração de renda sobre a redução da pobreza nos estados brasileiros", Anais do Encontro Nacional da Economia, Porto Seguro.

Marinho, E. y J. Araújo (2010), "Pobreza e o sistema de seguridade social rural no Brasil”, Revista Brasileira de Economia, vol. 64, № 2.

Marinho, E., F. Linhares y G. Campelo (2011), "Os programas de transferência de renda do governo impactam a pobreza no Brasil?", Revista Brasileira de Economia, vol. 65, № 3.

Menezes, T.A. y R.F. Pinto (2005), "É preciso esperar o bolo crescer, para depois repartir?", Anais do VIII Encontro Regional de Economia, Fortaleza.

Netto Júnior, J.L. da S. y E. Figueiredo (2014), "Crescimento pró-pobre no Brasil e nas regiões no período de 1987-2007: uma abordagem não paramétrica”, Planejamento e Políticas Públicas, № 42.

Ravallion, M. (1997), “Can high-inequality developing countries escape absolute poverty?", Economic Letters, vol. 56, № 1, Amsterdam, Elsevier.

(2004), "Pro-poor growth: a primer", Policy Research Working Papers, № 3242, Washington, D.C., Banco Mundial.

Ravallion, M. y S. Chen (2003), "Measuring pro-poor growth", Policy Research Working Paper, № 2666, Washington, D.C., Banco Mundial. (1997), "What can new survey data tell us about recent changes in distribution and poverty?", World Bank Economic Review, vol. 11, № 2, Washington, D.C., Banco Mundial.

Ribas, R.P., A.F. Machado y A.B. Golgher (2006), "Fluctuations and persistence in poverty: a transientchronic decomposition model for pseudo-panel data", Texto para Discussão, № 289, Universidad Federal de Minas Gerais.

Rocha, S. (2006), Pobreza no Brasil. Afinal de que se trata?, Río de Janeiro, FGV Editora.

Rodrik, D. (2000), "Growth versus poverty reduction: a hollow debate", Finance \& Development, vol. 37, $N^{\circ} 4$, Washington, D.C., Fondo Monetario Internacional.

Stewart, F. (2000), Distribuição de renda e desenvolvimento, Brasilia, NEAD.

Windmeijer, F. (2005), "A finite sample correction for the variance of linear efficient two-step GMM estimators", Journal of Econometrics, vol. 126, №1, Amsterdam, Elsevier. 